\title{
Dilution Effects in Two-dimensional Quantum Orbital System
}

\author{
Takayoshi Tanaka and Sumio Ishihara \\ Department of Physics, Tohoku University, Sendai 980-8578, Japan
}

(Dated: December 28, 2018)

\begin{abstract}
We study dilution effects in a Mott insulating state with quantum orbital degree of freedom, termed the two-dimensional orbital compass model. This is a quantum and two-dimensional version of the orbital model where the interactions along different bond directions cause frustration between different orbital configurations. A long-range correlation of a kind of orbital at each row or column, termed the directional order, is studied by means of the quantum Monte-Carlo method. It is shown that decrease of the ordering temperature due to dilution is much stronger than that in spin models. Quantum effect enhances the effective dimensionality in the system and makes the directional order robust against dilution. We discuss an essential mechanism of the dilute orbital systems.
\end{abstract}

PACS numbers: 71.10.-w, 71.23.-k, 75.30.-m

Recently, there is much effort to reveal impurity effects on correlated electron systems. One of the famous examples is the non-magnetic impurity doping into the lowdimensional quantum-magnets, such as the spin-Peierls systems and even-leg spin ladders, where the antiferromagnetic (AF) long-range order (LRO) is induced by breaking the quantum spin-singlet correlation.

Randomly dilute correlated electron systems with orbital degree of freedom is another class of disordered systems. Since this degree of freedom corresponds to anisotropy of the electronic clouds, the orbital interaction in a solid is essentially directional. That is to say, Hamiltonian explicitly depends on the bond direction unlike spin models [1, 2]. There are competition and frustration between the different-bond interactions which reduce the effective dimensionaliry in the system. Dilution effects in such a unique system have been studied experimentally in $\mathrm{KCu}_{1-x} \mathrm{Zn}_{x} \mathrm{~F}_{3}[3]$. One of the doubly degenerate $e_{g}$ orbitals in a $\mathrm{Cu}$ ion is occupied by a hole and exhibits $\mathrm{LRO}$ in $\mathrm{KCuF}_{3}$. With substitution of $\mathrm{Cu}$ by $\mathrm{Zn}$, the orbital ordering temperature $T_{\mathrm{OO}}$ rapidly decreases and disappears around $x=0.5$. This decrease of $T_{\mathrm{OO}}$ is much stronger than that in dilute magnets and in the percolation theory.

This experimental observation was well explained by the calculation based on the orbital Hamiltonian [5]:

$$
\mathcal{H}=2 J \sum_{i, l}\left(\mathbf{T}_{\mathbf{r}_{i}} \cdot \hat{m}^{l}\right)\left(\mathbf{T}_{\mathbf{r}_{i}+\hat{l}} \cdot \hat{m}^{l}\right) \varepsilon_{\mathbf{r}_{i}} \varepsilon_{\mathbf{r}_{i}+\hat{l}},
$$

with a factor $\varepsilon_{\mathbf{r}_{i}}$ taking one for $\mathrm{Cu}$ and zero for $\mathrm{Zn}$, and the bond direction $l$. Here $\mathbf{T}_{\mathbf{r}_{i}}$ is the pseudo-spin (PS) operator at site $i$, and $\hat{m}^{l}$ is a unit vector in the $T^{x}-T^{z}$ plane. When we take $\hat{m}^{l}=\left[\sin \left(\frac{2 n_{l}}{3} \pi\right), \cos \left(\frac{2 n_{l}}{3} \pi\right)\right]$ with $\left(n_{x}, n_{y}, n_{z}\right)=(2,1,0)$, this model represents the interaction between the $e_{g}$ orbitals. Essence of the orbital dilution is frustration between the different-bond interactions and a macroscopic orbital degeneracy in the classical ground state due to the interactions.

In this paper, we study dilution effects in the twodimensional and quantum version of Eq. (11) with $\hat{m}^{x}=$
$[1,0]$ and $\hat{m}^{z}=[0,1]$, termed the quantum orbitalcompass model (OCM) 6, 7]. This is a minimal orbital model representing the directional and frustrated interaction between orbitals. One of the major different points from the $e_{g}$-orbital model is that the Hamiltonian is invariant under a certain local transformations of the PS operator. Due to this symmetry, degeneracy of the orbital state is more serious than that in the $e_{g}$-orbital model, and the conventional site-diagonal order is not expected. Instead, the long-range correlation for one of the PS components appears along the vertical or horizontal direction in a square lattice, termed the directional order (DO). Dilution effects in DO are investigated by means of the quantum Monte-Carlo (QMC) method in addition to some rigorous analytical considerations. We, in particular, focus on the quantum effects in the orbital dilution. It is shown that quantum fluctuation from the classical DO state enhances the effective dimensionality in the system and makes DO robust against dilution.

The explicit form of OCM is given in a two-dimensional square lattice as

$$
\mathcal{H}=2 J \sum_{i, l=(x, z)} T_{\mathbf{r}_{i}}^{l} T_{\mathbf{r}_{i}+\hat{l}}^{l} \varepsilon_{\mathbf{r}_{i}} \varepsilon_{\mathbf{r}_{i}+\hat{l}}
$$

Since the sign of the interaction $J$ is gauged away by rotating $\mathbf{T}_{\mathbf{r}_{i}}$ with respect to the $y$ axis, we take it negative. This Hamiltonian without dilution $\left(\varepsilon_{\mathbf{r}_{i}}=1\right.$ for $\forall i$ ) is invariant under the following two-symmetry operations [7, 8]; (i) The global four-fold symmetry: the pseudo-spins at all sites and a crystal lattice are rotated by $\pi / 2$, simultaneously, with respect to the $y$ axis. (ii) The local symmetry at each column and row: the $z(x)$ component of all PSs at each column (row) along the $z(x)$ axis are flipped, i.e. $T_{r_{x}, r_{z}}^{z(x)} \rightarrow-T_{r_{x}, r_{z}}^{z(x)}$ for each $r_{x}\left(r_{z}\right)$. In addition, in the classical ferromagnetic-like ground state, there is the following continuous symmetry: (iii) the global PS direction is arbitrary in the $T^{x}-T^{z}$ plane.

Let us explain in more detail the symmetry (ii) which is unique in OCM. This symmetry operation at a row 
$r_{z}$ is done by the operator $P_{r_{z}}\left(\equiv \prod_{r_{x}} \sigma_{r_{x}, r_{z}}^{z}\right)$, with the Pauli matrices $\sigma_{\mathbf{r}}$, as $P_{r_{z}}^{-1} \sigma_{r_{x}, r_{z}}^{x} P_{r_{z}}=-\sigma_{r_{x}, r_{z}}^{x}$ and $P_{r_{z}}^{-1} \sigma_{r_{x}, r_{z}}^{z} P_{r_{z}}=\sigma_{r_{x}, r_{z}}^{z}$. On an equal footing, the symmetry operation at a column $r_{x}$ is done by the operator $Q_{r_{x}}=\prod_{r_{z}} \sigma_{r_{x}, r_{z}}^{x}$. The operators $P_{r_{z}}$ and $Q_{r_{x}}$ commute with $\mathcal{H}$, and $P_{r_{z}}$ s commute with each other as well as $Q_{r_{x}}$ s, but $\left[Q_{r_{x}}, P_{r_{z}}\right] \neq 0$. The energy eigen-values are characterized by the eigen-value set of $P_{r_{z}} \mathrm{~s}\left(p_{1}, \cdots, p_{L}\right)$ or that of $Q_{r_{x}} \mathrm{~s}\left(q_{1}, \cdots, q_{L}\right)$ in a $L \times L(=N)$ square lattice. That is, at least, there are the $L$ conserved quantities. Since $P_{r_{i}}^{2}=1$ and $P_{r_{i}}^{\dagger}=P_{r_{i}}, p_{i}= \pm 1$. It is easily shown that the conventional site-diagonal order parameter $N^{-1} \sum_{\mathbf{r}}\left\langle\mathbf{T}_{\mathbf{r}}\right\rangle$ is not invariant under this local-symmetry operation. According to the generalized Elitzur's theorem, it is shown that the site-diagonal orbital LRO does not occur [9]. Instead, a certain kind of LRO, termed the directional order, appears. This is a long-range correlation of $T^{z}\left(T^{x}\right)$ at each column (row), and corresponds to a breaking of the global fourfold symmetry (i) explained above. This nematic-like order has been studied by means of the correlation function $C_{r_{x}}^{z z} \equiv \lim _{r_{z} \rightarrow \infty}\left\langle T_{r_{x} 0}^{z} T_{r_{x} r_{z}}^{z}\right\rangle$ at zero temperature 8] and $q=N^{-1} \sum_{\mathbf{r}}\left(T_{\mathbf{r}}^{z 2}-T_{\mathbf{r}}^{x 2}\right)$ in the classical OCM [6] We introduce, as an order parameter of DO, a more convenient form $D$ defined by

$$
\begin{aligned}
D & =N^{-1}(1-x)^{-1} \\
& \times \sum_{\mathbf{r}}\left(T_{\mathbf{r}}^{z} T_{\mathbf{r}+\hat{\mathbf{z}}}^{z} \varepsilon_{\mathbf{r}} \varepsilon_{\mathbf{r}+\hat{\mathbf{z}}}-T_{\mathbf{r}}^{x} T_{\mathbf{r}+\hat{\mathbf{x}}}^{x} \varepsilon_{\mathbf{r}} \varepsilon_{\mathbf{r}+\hat{\mathbf{x}}}\right) .
\end{aligned}
$$

This parameter $D$ indicates the correlation of $T^{z}$ along the $z$ axis or that of $T^{x}$ along $x$. Unlike $q$, this is not a constant for the quantum PS operators. Since $D$ commutes with $P_{r_{z}}$ and $Q_{r_{x}}$, being different from $\mathbf{T}_{\mathbf{r}}$, it is possible for $\langle D\rangle$ to be finite.

To examine OCM numerically with and without vacancies, we adopt QMC method in a finite-size cluster. There is no negative-sign problem in OCM. The QMC calculations have been performed in a square lattice of $L \times L$ sites $(L=14 \sim 18)$ with the periodic-boundary condition. We chose the Trotter number $n$ to be $12 \sim 22$, and extrapolate the calculated results to $n=\infty$. At low temperatures of $T<0.04 \mathrm{~J}$, we perform the simulation with $n=60 \sim 72$. We take, at maximum, 10,000MC steps for measurement after $3,000 \mathrm{MC}$ steps for thermalization. Physical quantities are averaged over 20 60MC samples at each parameter set. We have checked by MC calculations with $10^{6} \mathrm{MC}$ steps, which is much longer than the integrated autocorrelation time determined by the binning analyses, that the order parameter values in the present calculation are reliable within numerical errors. The ordering temperature $\left(T_{\mathrm{DO}}\right)$ of $\mathrm{DO}$ is estimated from the finite-size scaling analyses of the Binder cumulant for $D$ defined by $Q=1-\frac{\left\langle D^{4}\right\rangle}{3\left\langle D^{2}\right\rangle^{2}}[6,10]$. At $T=T_{\mathrm{DO}}$, a value of $Q$ should be size independent.

First we show the numerical results in OCM without
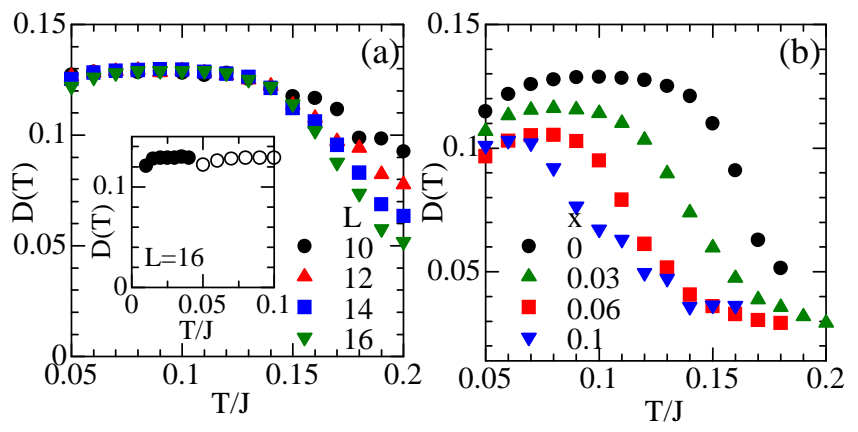

FIG. 1: (color online). (a) Temperature dependence of the directional order parameter $\bar{D}(T)$ at $x=0$ for various system sizes $L$. The inset shows $\bar{D}(T)$ at low temperatures obtained in the calculation with the large Trotter numbers (filled circles). Open circles are the same data with those in the main panel. (b) $\bar{D}(T)$ for various impurity concentrations.

vacancies. Temperature dependence of $\sqrt{\left\langle D^{2}\right\rangle}(\equiv \bar{D}(T))$ for several $L$ is presented in Fig. 1(a). Around $T / J=$ $0.17, \bar{D}(T)$ grows with decreasing $T$, and is saturated to about 0.13 below $T / J=0.12$. With increasing $L$, this dependence becomes steep, but the saturated value of $\bar{D}(T)$ at low temperatures does not change much. Below $T / J=0.07, \bar{D}(T)$ seems to decrease. We have performed careful numerical calculations with the larger Trotter numbers $n=60 \sim 72$. As shown in the inset of Fig. $1(\mathrm{a}), \bar{D}(T)$ takes about 0.125 down to $T / J=0.015$. Therefore, the reduction of $\bar{D}(T)$ in low temperatures is an artifact due to the small Trotter number. Temperature dependence of the Binder cumulant $Q$ is presented in Fig. 2. The $Q-T$ curves for the several system size $L$ cross around $T / J=0.15$ corresponding to the temperature where $\bar{D}(T)$ grows. We have also calculated the susceptibility $\chi_{D}$ of $D$ and the specific heat $C$. Both of them show peak structures around $T / J=0.15$, which become steep with increasing $L$. We conclude in the present calculation that DO is realized in the quantum OCM and $T_{\mathrm{DO}}=0.150 \pm 0.003$. It is noted that the saturated value of $\bar{D}$ at low temperatures is about a half of the classical value of $1 / 4$. This reduction is much stronger than that in the quantum AF Heisenberg model with $S=1 / 2$ in a square lattice where the saturated staggered moment is about $60 \%$ of $1 / 2$ 11. We also calculate the site-diagonal order parameter $\bar{M} \equiv \sqrt{\left\langle\mathbf{M}^{2}\right\rangle}$ with $\mathbf{M}=N^{-1} \sum_{\mathbf{r}} \mathbf{T}_{\mathbf{r}}$. The calculated $\bar{M}$ is almost temperature independent and its magnitude decreases with increasing $L$. This is, the site-diagonal order does not tend to occur because of the local symmetry (ii), mentioned previously. This situation is in contrast to the $e_{g}$-orbital model where the site-diagonal order is realized by the order-by-disorder mechanism.

Now we present the dilution effects in OCM. $\bar{D}(T)$ for 


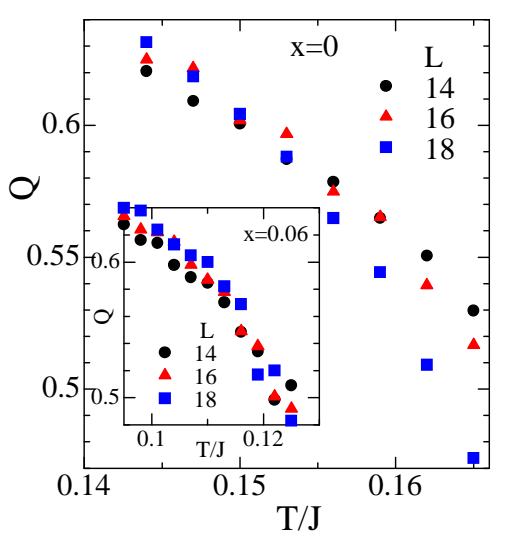

FIG. 2: (color online). Temperature dependence of the Binder cumulant $Q$ at $x=0$ for various system sizes $L$. Inset shows temperature dependence of $Q$ at $x=0.06$.

several $x$ are shown in Fig. 1(b). $\bar{D}(T)$ gradually increases with decreasing $T$ and is saturated, for example, to about 0.1 at $x=0.06$. The temperature where $\bar{D}(T)$ rises up is reduced with doping. The saturated value of $\bar{D}(T)$ becomes small by doping in spite of a factor $(1-x)^{-1}$ in Eq. (31). This phenomenon was also observed in the dilute $e_{g}$-orbital model [5] and will be discussed in more detail later. The analyses for $Q$ work well at $x=0.06$, as shown in the inset of Fig. 2 , where $T_{\mathrm{DO}} / J$ is identified to be $0.113 \pm 0.005$. We have checked that, at $x=0.25$, the $Q-T$ curves for several $L$ do not cross with each other down to $T=0.01 \mathrm{~J}$.

The main results in the present work, the $x$ dependence of $T_{\mathrm{DO}}$ in the quantum OCM, are shown in Fig. 3. For comparison, we plot the ordering temperature $T_{\mathrm{CL}}$ of $\mathrm{DO}$ in the classical OCM and the Curie temperature $T_{\text {Ising }}$ in the $S=1 / 2$ Ising model in a square lattice. $T_{\mathrm{DO}}$ decreases monotonically with $x$ and seems to take zero around $x=0.15\left(\equiv x_{c}\right)$. It is worth to note that $x_{c}$ is much smaller than the percolation threshold 0.41 in a square lattice [4]. The decrease of $T_{\mathrm{DO}}$ is much stronger than that in $T_{\text {Ising }}$, but is weaker than $T_{\mathrm{CL}}$. In other words, quantum effect makes DO robust against dilution.

To understand the unusual dilution effects in the quantum OCM, we present a rigorous treatment of the dilute OCM in two-coupled chains along the $z$ axis, that is, a two-leg ladder OCM. As mentioned previously, the energy eigen-states are classified by the eigen-value set $\left(p_{1}, \cdots, p_{L}\right)$ of the operator $P_{r_{z}}=\sigma_{r_{L} r_{z}}^{z} \sigma_{r_{R} r_{z}}^{z}$ where $r_{R}\left(r_{L}\right)$ indicates the $x$ coordinates of the right (left) chain in the ladder. We consider one of the two-fold ground state with $p_{1}=\cdots=p_{L}=1$ being degenerate with the state characterized by $p_{1}=\cdots=p_{L}=-1$. In a row $r_{z}$ of the ladder, the eigen states are $\left|T_{r_{L} r_{z}}^{z} T_{r_{R} r_{z}}^{z}\right\rangle=|\uparrow \uparrow\rangle$ and $|\downarrow \downarrow\rangle$, or their linear combinations $| \pm\rangle \equiv \frac{1}{\sqrt{2}}(\mid \uparrow \uparrow$ \rangle$\pm|\downarrow \downarrow\rangle)$. There are relations $\left(T_{r_{L} r_{z}}^{x} T_{r_{R} r_{z}}^{x}\right)| \pm\rangle= \pm \frac{1}{4}| \pm\rangle$,

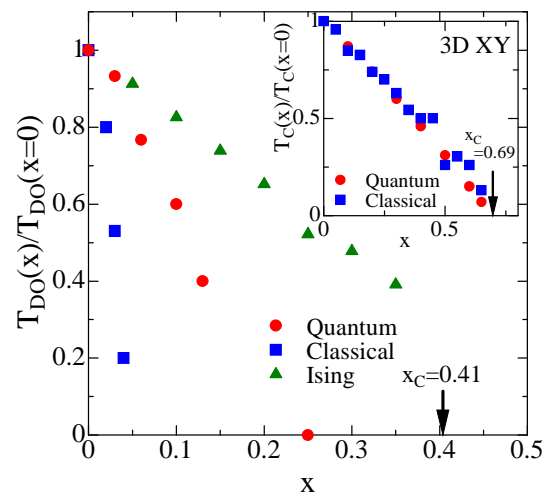

FIG. 3: (color online). Impurity concentration $x$ dependence of the ordering temperature. Closed circles are for $T_{\mathrm{DO}}$ in the quantum OCM, and closed squares are for $T_{\mathrm{CL}}$ in the classical OCM. For comparison, the ordering temperature in the Ising model (triangles) are also plotted. Bold arrow indicates the percolation threshold $x_{c}$ in a square lattice. The inset shows the ordering temperature $T_{\mathrm{C}}$ in the quantum XY model (circles) and that in the classical one (squares) in a three-dimensional cubic lattice. System size is chosen to be $N=23^{3}$ at maximum. The continuous imaginary-time algorithm is utilized.

$T_{r_{L} r_{z}}^{z}| \pm\rangle=\frac{1}{2}|\mp\rangle$ and $T_{r_{R} r_{z}}^{z}| \pm\rangle=\frac{1}{2}|\mp\rangle$. Therefore, within the subspace of $p_{1}=\cdots=p_{L}=1$, we introduce new pseuso-spin operators $\mathbf{S}_{r_{z}}$ and show correspondences $\left(T_{r_{L} r_{z}}^{x} T_{r_{R} r_{z}}^{x}\right) \rightarrow \frac{1}{2} S_{r_{z}}^{z}$ and $T_{r_{L}\left(r_{R}\right) r_{z}}^{z} \rightarrow S_{r_{z}}^{x}$. The state $|+\rangle(|-\rangle)$ is the up- (down-) eigen state of the operator $S_{r_{z}}^{z}$. As a result, OCM in this subspace is mapped onto the transverse-Ising model in a single chain [13]: $\mathcal{H}_{\text {eff }}=-4 J \sum_{r_{z}} S_{r_{z}}^{x} S_{r_{z}+1}^{x}-J \sum_{r_{z}} S_{r_{z}}^{z}$. Now one vacancy is introduced at a site $\left(r_{L}, r_{z}^{0}\right)$. Since, in the row $r_{z}^{0}$, we have $P_{r_{z}^{0}}=\sigma_{r_{R} r_{z}^{0}}^{z}$, the energy eigen states in this row, $|-\uparrow\rangle$ and $|-\downarrow\rangle$, belong to the different subspace, and $T_{r_{R} r_{z}^{0}}$ is replaced by a $C$-number in each subspace. Thus, OCM in the two-leg ladder with one vacancy is denoted by the following model:

$$
\begin{aligned}
\mathcal{H}_{\text {eff }} & =-4 J \sum_{r_{z}}^{\prime} S_{r_{z}}^{x} S_{r_{z}+1}^{x}-J \sum_{r_{z} \neq r_{z}^{0}} S_{r_{z}}^{z} \\
& \pm J\left(S_{r_{z}^{0}+1}^{x}+S_{r_{z}^{0}-1}^{x}\right),
\end{aligned}
$$

where $\sum_{r_{z}}^{\prime}$ indicates a sum of $r_{z}$ except for $r_{z}^{0}$ and $r_{z}^{0}-1$. Signs + and - in the third term correspond to the states $|-\uparrow\rangle$ and $|-\downarrow\rangle$ at the site $\left(r_{R}, r_{z}^{0}\right)$, respectively. This model is the two transverse-Ising chains where the external field $\pm J$ along the $S^{x}$ axis is applied at edges of the chains, i.e. the $r_{z}^{0}+1$ and $r_{z}^{0}-1$ sites . In the original language, this field acts on $T_{r_{R}\left(r_{L}\right), r_{z}^{0}+1}^{z}$ and $T_{r_{R}\left(r_{L}\right), r_{z}^{0}-1}^{z}$, and enhances the correlation $\left\langle T_{\mathbf{r}_{i}}^{z} T_{\mathbf{r}_{j}}^{z}\right\rangle$ around the vacancy. The above analyses accord with an intuitive picture for roles of vacancy in OCM; due to the directional interaction in the model, orbitals near the vacant site ad- 


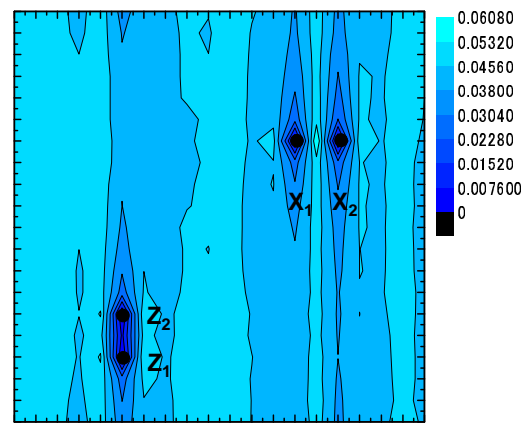

FIG. 4: (color online). Contour map of local static correlation function $C^{z z}\left(\mathbf{r}_{i}\right)$ in a $20 \times 20$ site cluster. Four vacancies $\mathrm{X}_{1(2)}$ and $\mathrm{Z}_{1(2)}$ are represented by black circles. Temperature is chosen to be $T / T_{\mathrm{DO}}(x=0)=1.27$.

just their directions to gain the exchange energy. In other words, vacancy tends to fix the orbital direction around it.

Being based on the above picture, we discuss the difference between $T_{\mathrm{DO}}$ and $T_{\text {Ising }}$ in Fig. 3. Consider a situation in a two-dimensional square lattice where two vacancies are located closely in a same row, e.g. at sites $\left(r_{x}-1, r_{z}\right)$ and $\left(r_{x}+1, r_{z}\right)$. This probability is of the order of $x$. Since PS between the vacancies has the Ising-type interaction $T_{r_{x} r_{z}}^{z}\left(T_{r_{x} r_{z}+1}^{z}+T_{r_{x} r_{z}-1}^{z}\right)$, the correlation of $T^{z}$ is enhanced around the vacancies, as suggested above. On an equal footing, the correlation of $T^{x}$ is enhanced around the vacancies located closely in a same column. It is expected that a certain number of domains/patches, where $T^{z}$ or $T^{x}$ correlation is enhanced, coexist in a system. Competition between such kinds of domains reduces the order parameter $\bar{D}(T)$ and the ordering temperature $T_{\mathrm{DO}}$. To confirm this picture, we calculate the localstatic PS correlation function around the impurity defined by $C^{z z}\left(\mathbf{r}_{i}\right)=\frac{1}{N(1-x)} \sum_{j}\left\langle T_{\mathbf{r}_{\mathbf{i}}}^{z} T_{\mathbf{r}_{\mathbf{j}}}^{z}\right\rangle$ (Fig. 4). Temperature is chosen to be $T / T_{\mathrm{DO}}(x=0)=1.27$. Two pairs of vacancies introduced in a $20 \times 20$ site cluster are denoted by $X_{1(2)}$ and $Z_{1(2)}$ in the figure. It is clearly shown that $C^{z z}\left(\mathbf{r}_{i}\right)$ is enhanced between the impurities $\mathrm{X}_{1}$ and $\mathrm{X}_{2}$, and almost vanishes between $Z_{1}$ and $Z_{2}$. These results are not seen in dilute spin models.

Next we focus on quantum effects in dilution, i.e. discrepancy between $T_{\mathrm{DO}}$ and $T_{\mathrm{CL}}$. In the classical DO state at $T=0$, the system is considered to be decoupled into the independent Ising chains along the $x$ or $z$ axis, although DO is the two-dimensional order where the four-fold symmetry is broken. The rapid decrease of $T_{\mathrm{CL}}$ by dilution reflects the quasi-one dimensional nature of DO, and at finite temperatures, weak two-dimensionality is recovered due to the thermal fluctuation. The quantum fluctuation from the classical DO state brings about a coupling between the independent chains and increases effective dimensionality of DO even at low temperatures. This is a kind of resonant states between the independent chains and DO becomes robust against dilution. This remarkable quantum effect is unique in the orbital system; we compare the $x$ dependence of the Curie temperatures $T_{\mathrm{C}}$ in the quantum and classical $\mathrm{XY}$ models in a cubic lattice (the inset of Fig. 3). A magnitude of spin is chosen to be $1 / 2$ in the quantum model. It is seen that difference between the two are much smaller than that in OCM, and both $T_{\mathrm{C}}$ 's in the XY model seem to disappear at the percolation threshold $\left(x_{c}=0.69\right)$.

Before summarizing the results, we briefly discuss implications of the dilute $e_{g}$-orbital model. In our previous studies [5], we have suggested that a remarkable decrease of $T_{\mathrm{OO}}$ is related to the tilting of PS around impurities. Roles of this PS tilting become clearer by the present analyses of OCM. The orbital interaction in the $e_{g}$-orbital model is essentially of the Ising-type along one direction in a cubic lattice, and there is frustration between them, as well as OCM. Introduced vacancies break the local symmetry and release the frustration. According to vacancy configurations, a specific type of the orbital correlation is stabilized around vacancies. For example, when two vacancies are located closely at a same row along the $z$ axis, the orbital correlation of $d_{3 x^{2}-r^{2}} / d_{y^{2}-z^{2}}$ or $d_{3 y^{2}-r^{2}} / d_{z^{2}-x^{2}}$ are stabilized around them. Competition between these domains/patches with different PS correlations reduce the order parameter and may cause the glass-like phase.

In summary, we study the dilution effects in a quantum and two-dimensional version of the orbital model, i.e. the orbital compass model, where the vertical or horizontal correlation of a PS component, termed the directional order, is expected from the local symmetry. Reduction of the ordering temperature of DO is much stronger than that in the spin model. Quantum fluctuation increases the effective dimensionality of the system and makes DO robust against dilution. Competition between domains with different orbital configurations is essence of the dilute orbital system.

The authors thank to M. Matsumoto for several helpful discussion and providing calculation data for the inset in Fig. 3, and H. Matsueda for valuable comments. This work was supported by Grant-in-Aid for Scientific Research on priority Areas, JSPS KAKENHI (16340094, 16104005) and TOKUTEI "High Field Spin Science in 100T" (No.451, 18044001) from MEXT, NAREGI, and CREST. T. T. appreciates financial support from JSPS.

[1] S. Maekawa, et al., Physics of Transition Metal Oxides (Springer Verlag, Berlin, 2004), and references therein.

[2] K. I. Kugel and D. I. Khomskii, Sov. Phys. JETP 37, 725 (1973). 
[3] N. Tatami, S. Niioka, and Y. Murakami, (to be published).

[4] D. Stauffer, Phys. Rep. 54, 1 (1979).

[5] T. Tanaka, et al., Phys. Rev. Lett. 95, 267204 (2005).

[6] A. Mishra, et al., Phys. Rev. Lett. 93, 207201 (2004).

[7] Z. Nussinov, et al., Euro. Phys. Lett. 67, 990 (2004).

[8] J. Dorier, et al., Phys. Rev. B 72, 024448 (2005).
[9] C.D. Batista and Z. Nussinov, Phys. Rev. B. 72, 045137 (2005).

[10] K. Binder, Phys. Rev. Lett. 47, 693 (1981).

[11] E. Manousakis, Rev. Mod. Phys. 63, 1 (1991).

[12] R. B. Stinchcombe, J. Phys. C 13, 5565 (1980).

[13] B. Doucot, et al., Phys. Rev. B 71, 024505 (2005). 